\title{
Growth of Hybrid Inorganic/Organic Chiral Thin Films by Sequenced Vapor Deposition
}

Ortal Lidor-Shalev ${ }^{1}$, Reut Yemini ${ }^{1}$, Nicole Leifer ${ }^{2}$, Raju Nanda ${ }^{3}$, Aviv Tibi ${ }^{4}$, Ilana Perelshtein ${ }^{5}$, Efrat Shawat Avraham ${ }^{6}$, Yitzhak Mastai ${ }^{7}$ and Malachi Noked ${ }^{*}$

Department of Chemistry and the Institute for Nanotechnology and Advanced Materials Bar-Ilan University, Ramat-Gan 5290002, Israel.

*Malachi.Noked@biu.ac.il

HRSEM image of cross-section of Al\&LCys thin films deposited over Si wafer (shown in Figure SI1a) demonstrates a thickness of $6 \pm 1 \mathrm{~nm}$. Figure SI1b shows HRTEM image of Al\&LCys thin films prepared over Si wafer, supporting the dimensions of $6 \pm 0.5 \mathrm{~nm}$ thickness of Al\&LCys thin films. Energy-dispersive X-ray (EDAX) of Al\&LCys thin films (Figure SI1c) supports the presence of $\mathrm{Al}, \mathrm{O}, \mathrm{C}$ and $\mathrm{S}$. 
a

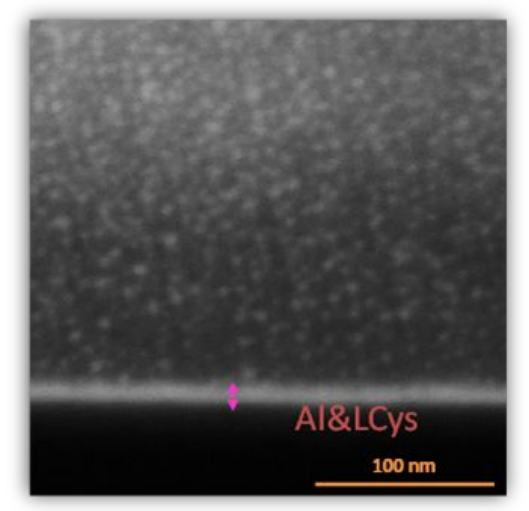

b

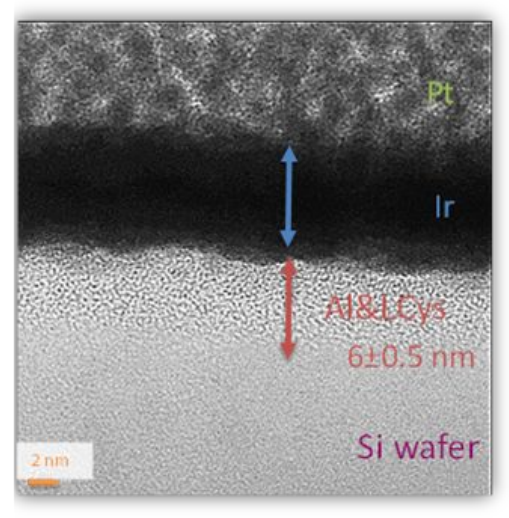

C

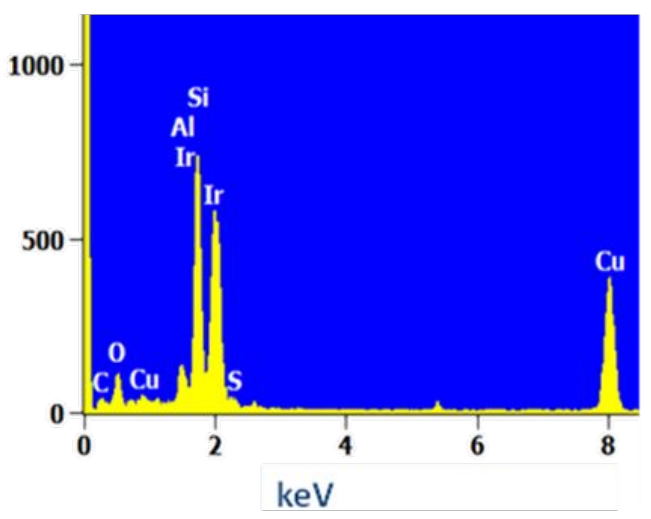

d

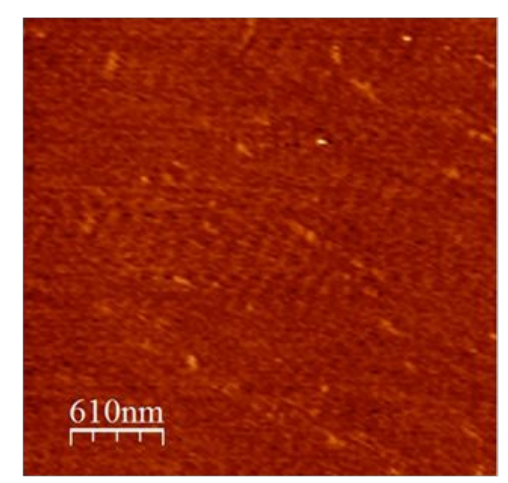

e

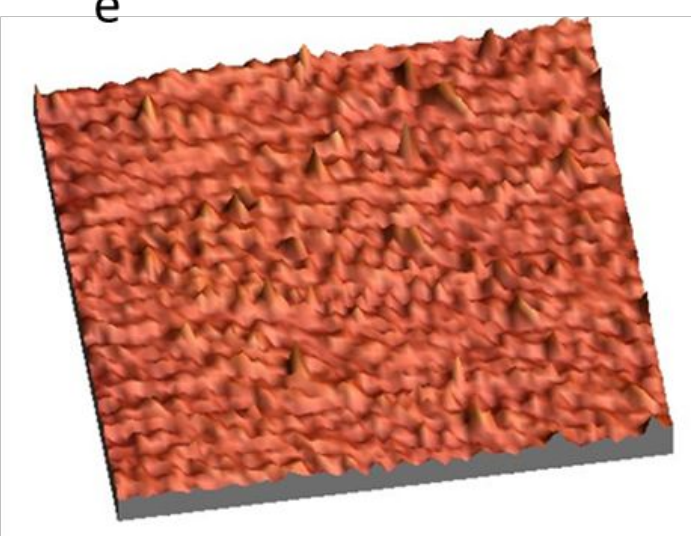

Figure SI 1. Structural study of Al\&LCys thin films. a, FIB cross section of AI\&LCys thin films prepared over Si wafer, coated with $\sim 6 \mathrm{~nm}$ Ir layer (to prevent charging effects), demonstrating a total thickness of $12 \pm 1 \mathrm{~nm}$ obtained after 50 SVD cycles. b, HRTEM image of Lamella cross-section of Al\&LCys thin films demonstrating thickness of $6 \pm 0.5 \mathrm{~nm}$. c, EDAX of AI\&LCys thin films deposited over Si wafer taken by HRTEM cross section of lamella (shown in b). d, 2D and e, 3D AFM images of AI\&LCys thin films obtained after 50 SVD cycles. 
a

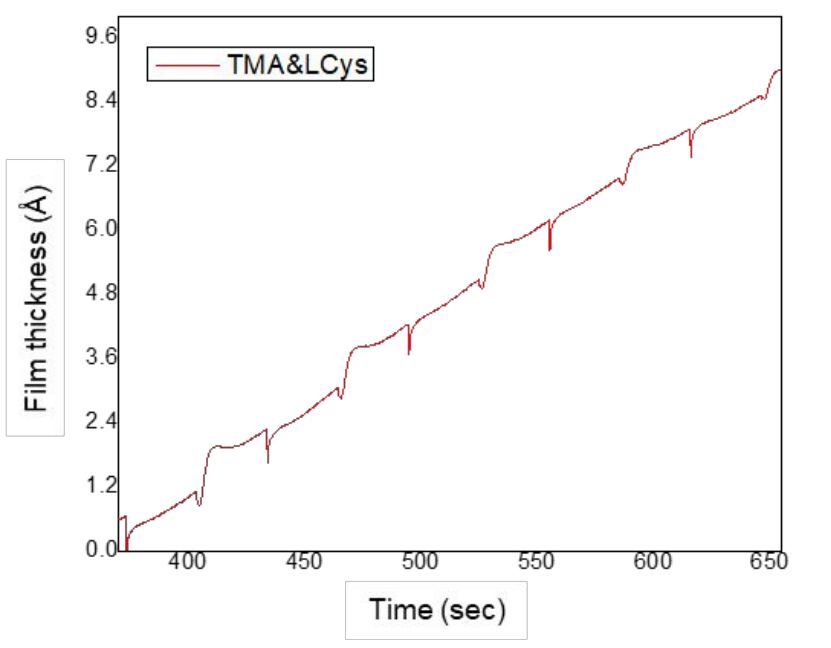

b

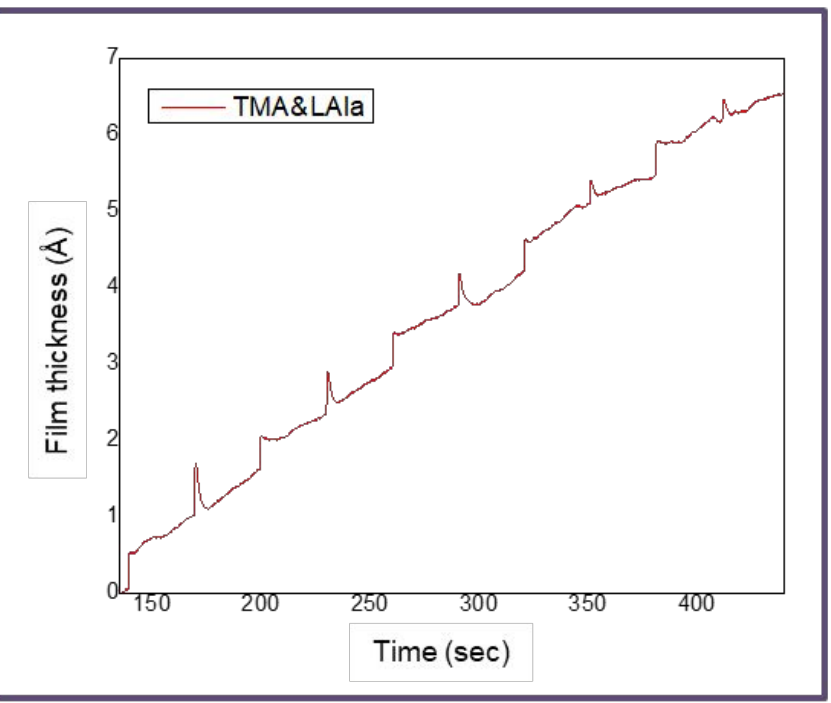

Figure SI 2. In-situ SVD-QCM profile of the thin films growth. a, QCM profile of 5 SVDcycles for the growth of AI\&LCys thin films, using alternating sequential pulses of TMA and LCys, b, QCM profile of 5SVD cycles for the growth of AI\&LAla thin films, using alternating sequential pulses of TMA and Lala.

For accurate thickness calculation using relevant model for QCM analysis, we need density of our material, since our film density is unknown, further analysis of QCM data is problematic. Hence, we calculated the thickness from the HRTEM images.

a

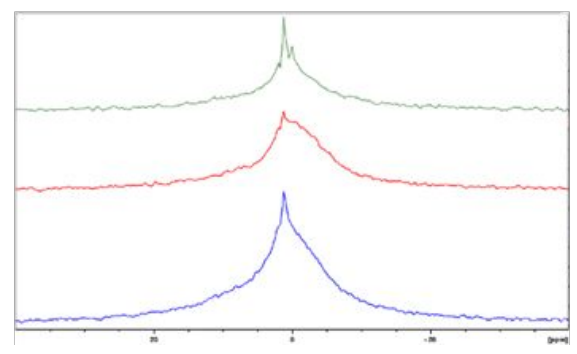

b

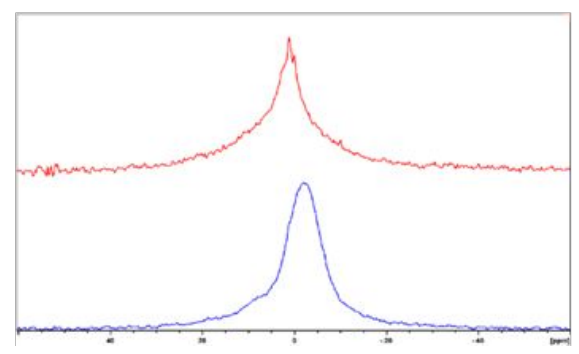

C

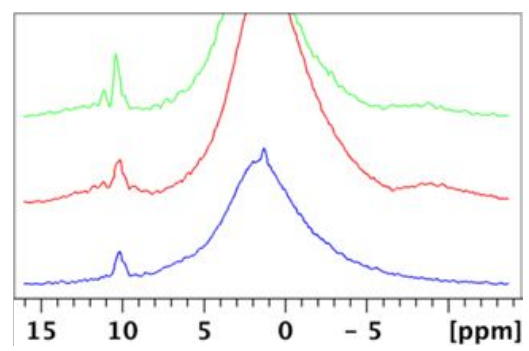

Figure SI 3. ${ }^{1} \mathrm{H}$ NMR spectra. a, ${ }^{1} \mathrm{H}$ Hahn echo MAS NMR of 1 cycle sample (blue, bottom), 1.5 cycle sample (red, middle), and 2 cycle sample (green, top). Spectra collected at $5 \mathrm{kHz}$ spinning speed after 6 rotor cycles. b, ${ }^{1} \mathrm{H}$ spectra of untreated $\mathrm{HC}$ (blue, bottom) and 40 alternating $\mathrm{ALD}$ cycles of $\mathrm{Al}_{2} \mathrm{O}_{3}$ and $\mathrm{H}_{2} \mathrm{O}$ (red, top). c, ${ }^{1} \mathrm{H}$ NOESY MAS NMR, positive row projection of data from 1 cycle sample (blue, bottom), 1.5 cycle sample (red, middle), and 2 cycle sample (green, top). 
a

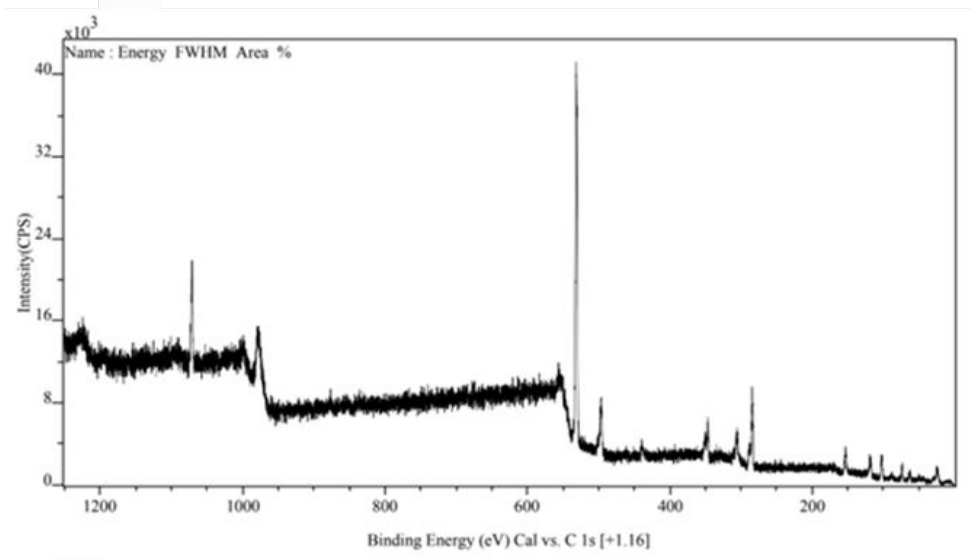

b

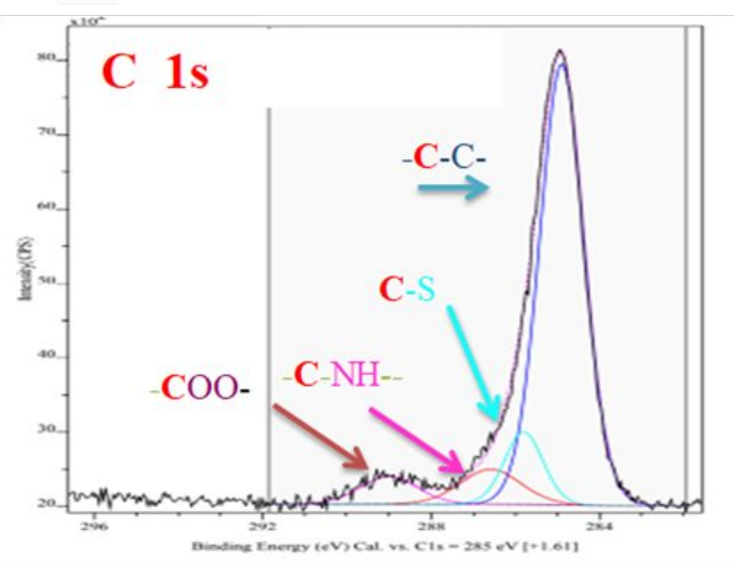

d

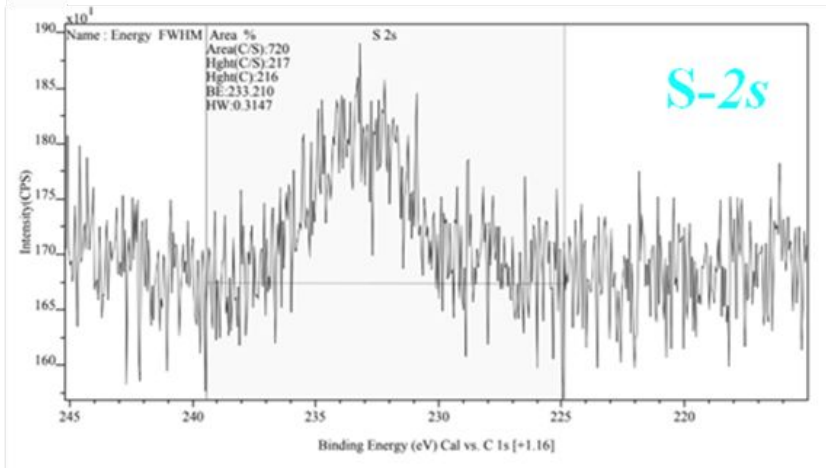

f

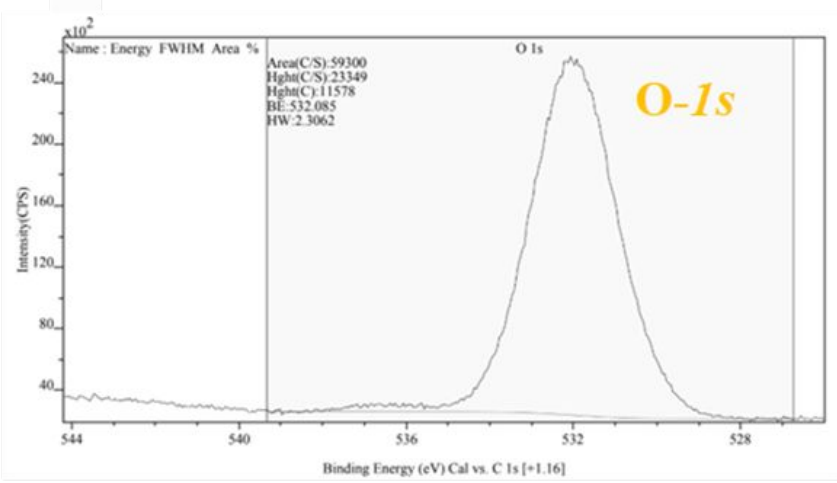

Figure SI 2. Elemental analysis of AI\&Lcys thin films deposited over Si wafer. a, XPS spectra. b, C-1s spectra and the deconvolution, c, N-1s spectra, d, S-2s spectra. e, Al-2p spectra, and f, O-1s spectra. 
Figure SI5 a-b shows the CD signals and enantioselectivity calculations of L- Phe and D-Phe before and after the adsorption experiments over $\sim 14 \mathrm{~nm}$ thin films of Al\&LAla. In this case, the thin films have shown $31.95 \%$ change in the CD signal of L-Phe enantiomer and $47.17 \%$ change in the CD signal of the D-Phe enantiomer, hence demonstrating enantioselectivity of $15.2 \%$ in favor of the D- Phe enantiomer.

All the enantiospecifity were calculated by

$$
100 * \frac{\Delta C D \text { of one enantiomer }}{C D \text { signal of its mother solution }}-100 * \frac{\Delta C D \text { of second enantiomer }}{C D \text { signal of its mother solution }}=\% \text { enantiospecifity }
$$

a

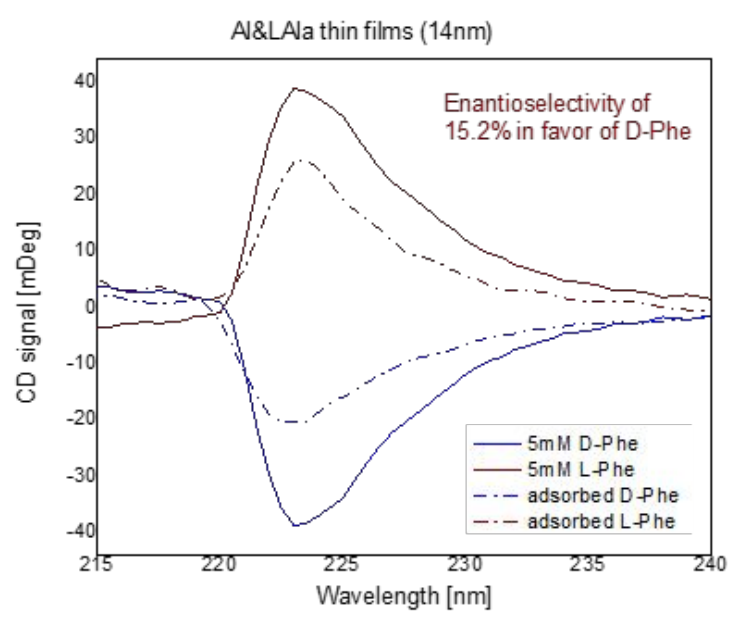

b

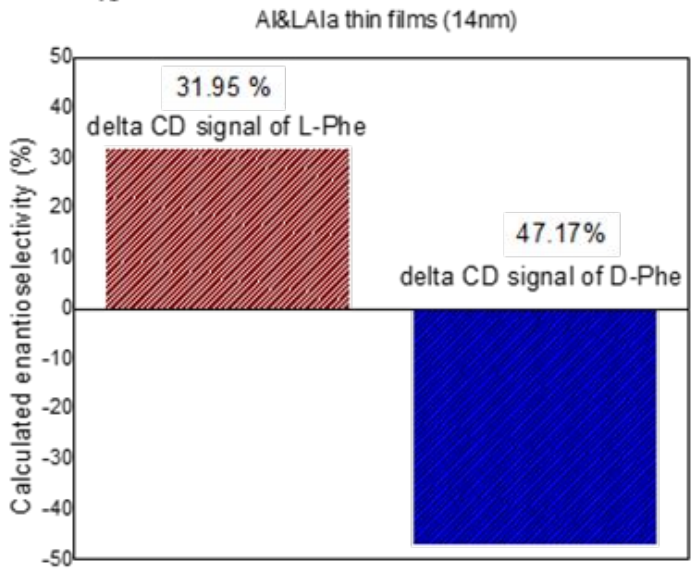

Figure SI 3. CD measurements of enantioselective adsorption experiments of D-Phe and L-Phe over AI\&LCys thin films. a, CD signals of $5 \mathrm{mM}$ aqueous solutions of L-Phe and D-Phe before and after the adsorption experiments over $14 \mathrm{~nm}$ thin films of AI\&LAla prepared over glass substrates, demonstrating $15.2 \%$ enantioselectivity in favor of $D-$ Phe. $\mathbf{b}$, Calculated $\triangle C D$ of $5 \mathrm{mM}$ D-Phe and L-Phe adsorbed over $14 \mathrm{~nm}$ Al\&LAla thin films, revealing 31.95\% change in the CD signal of L-Phe enantiomer and $47.17 \%$ change in the CD signal of the D-Phe enantiomer. 
a

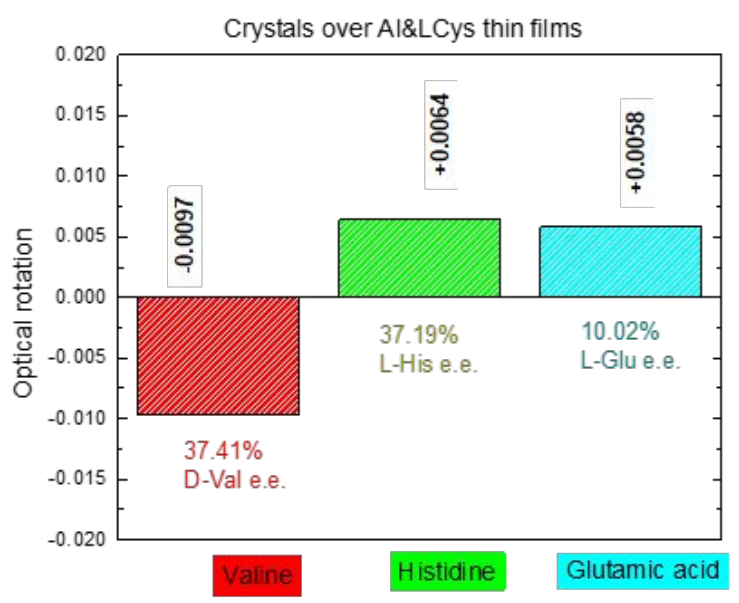

C

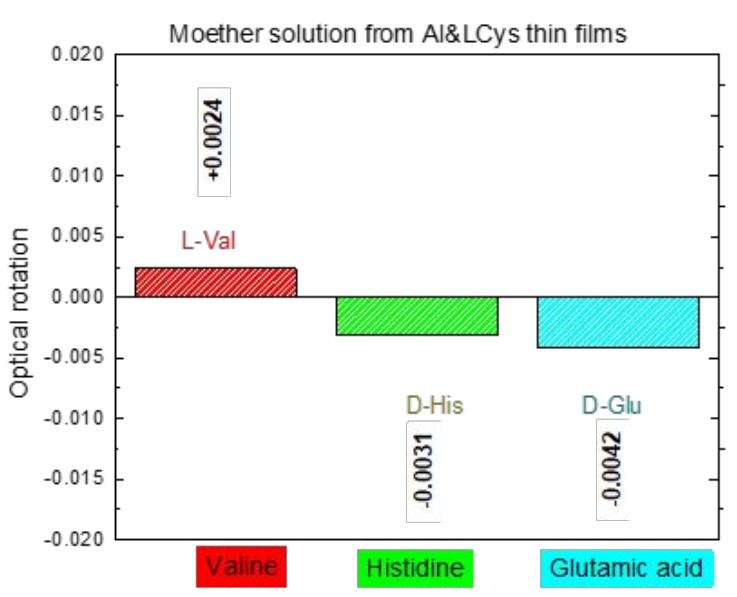

b
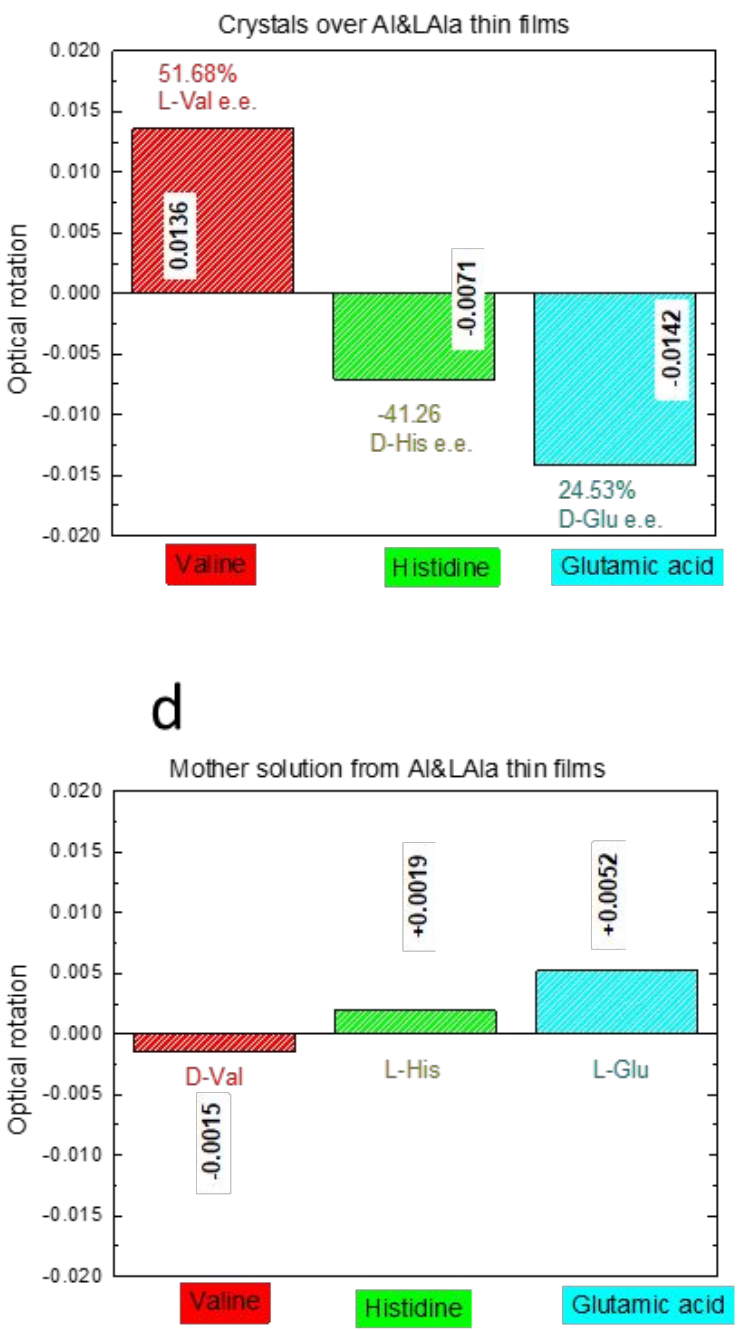

e

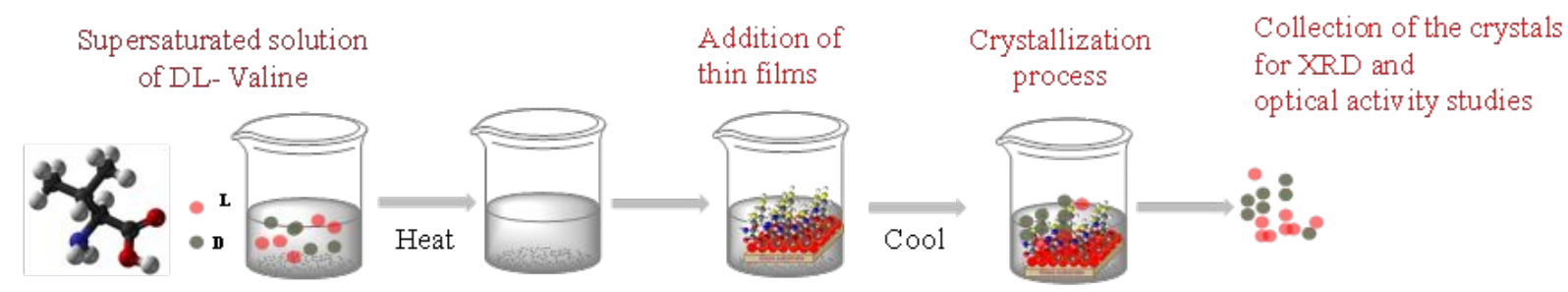

Figure SI 4. Polarimetry optical rotations measurements of enantioselective crystallization experiments after crystallization experiments over AI\&LCys and AI\&LAla thin films. a, optical rotations of Val, His, and Glu crystals crystalized over AI\&LCys thin films (12 nm thick over glass substrate). b, optical rotation of Val, His, and Glu crystals crystalized over AI\&LAla thin films (14 nm thick over glass substrate). c, optical rotations of the mother solution of a. d, the optical rotations of the mother solution of $\mathrm{c}$. e. scheme shows the concept of the crystallization process of racemic compound over chiral thin films. 


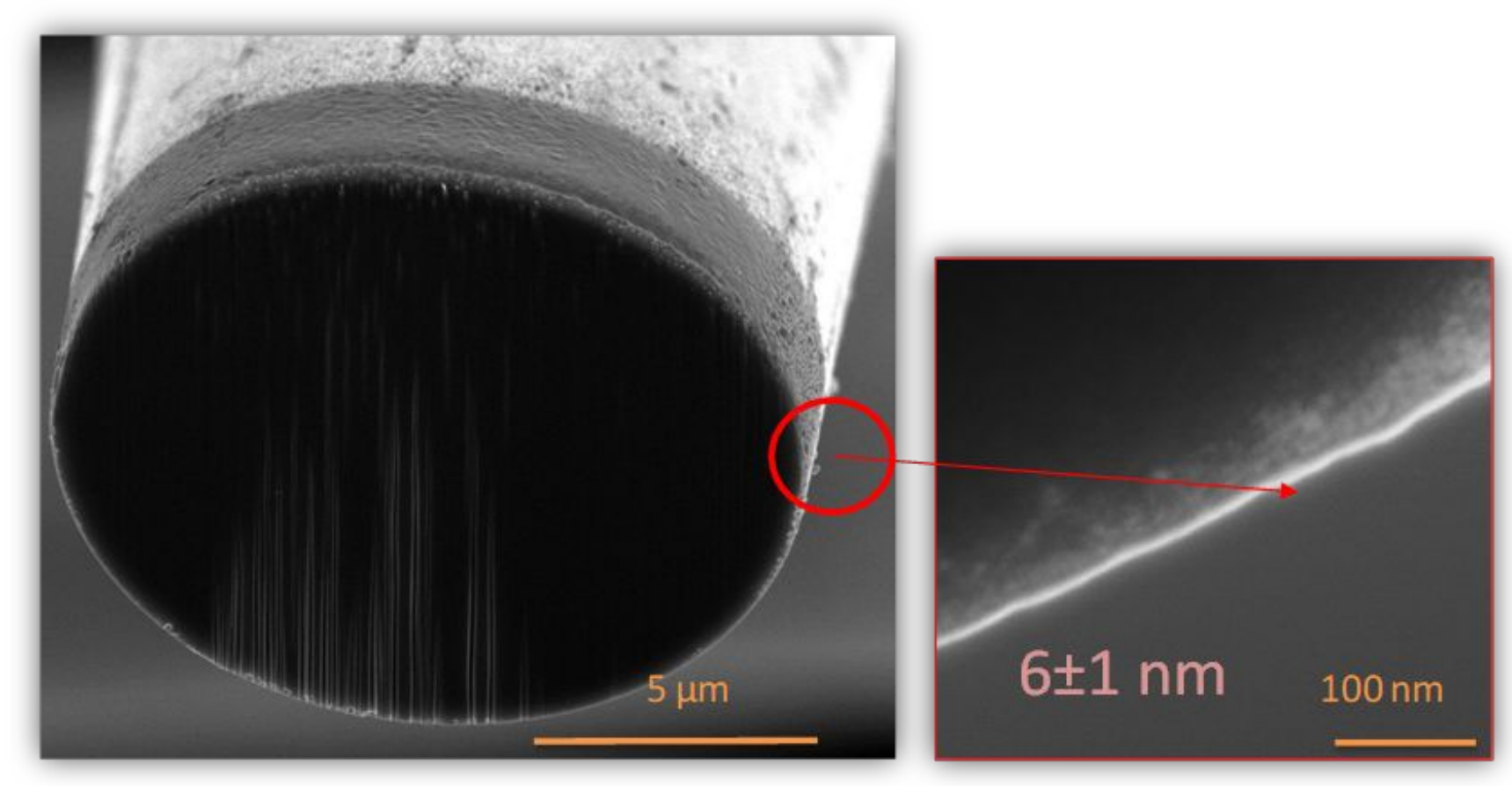

Figure SI 5. Cross sections study of Al\&LCys thin films. FIB cross section images of ACC coated with Al\&LCys, demonstrating a thickness of $\sim 6 \mathrm{~nm}$ layer. 
Al\&LAla thin films prepared over ACC have revealed a new CD signal that was assigned from the leftover solution and can be attributed to L-Phe enantiomer (Figure SI8a) caused by enantioselective adsorption of D-Phe over the thin films. This is sensible with the D-Phe and L-Phe adsorption experiments which have shown enantioselectivity of $16.53 \%$ in favor of the D-Phe (Figure SI8b-c).

a

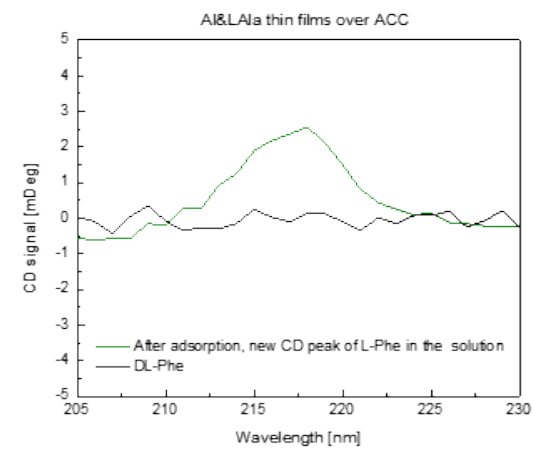

b

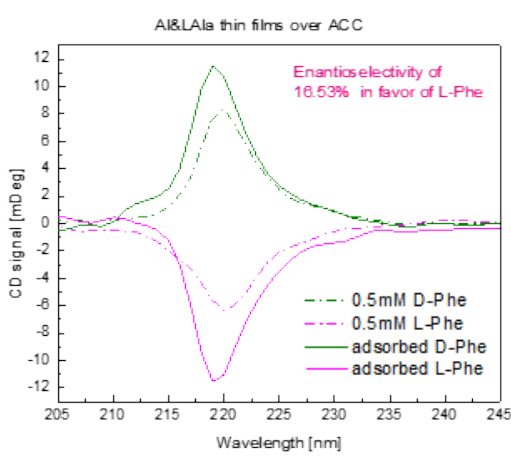

C

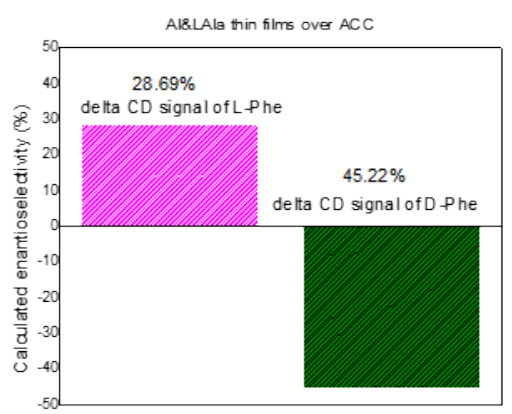

Figure SI 6. CD measurements of enantioselective adsorption experiments of Phe over Al\&LAla thin films. a, CD signals of DL-Phe before adsorption (black) and after 48 hours (green) adsorption over Al\&LAla thin films $\mathbf{b}$, CD signals of D-Phe and L-Phe before and after adsorption experiments, c, Calculated $\triangle \mathrm{CD}$ of L-Phe signals and D-Phe signals for enantioselectivity calculations. 


\section{Methods}

\section{Al\&LCys and Al\&LAla thin films growth:}

ALD Savannah S200 G2 system was used for the SVD process. The thin films were grown under 0.15 Torr of argon flow. To ensure complete removal of residual reactants and by-products in each step, a long purge time was used between every two precursor pulses. Expo mode was further used for enabling more exposure to the surface. For Al\&LCys and Al\&LAla thin films growth, the ALD chamber was preheated to $150^{\circ} \mathrm{C}$, the amino acid precursors were heated to $135^{\circ} \mathrm{C}$, and the $\mathrm{Al}$ precursor tri-methyl-aluminum $\left(\mathrm{Al}\left(\mathrm{CH}_{3}\right)_{3}\right.$, TMA) precursor was not heated. Each ALD cycle consists of 0.02 seconds of exposure to the TMA precursor followed by 10 seconds of argon purge, and then 0.0055 seconds exposure to the amino acid precursor, followed by 10 seconds of argon purge. For the expo mode, the valve of the chamber was closed after each pulse for 10 seconds. In the long-pulse mode, Each ALD cycle consists of 0.02 seconds of exposure to the TMA precursor followed by 30 seconds of argon purge, and then 0.0055 seconds exposure to the amino acid precursor, followed by 30 seconds of argon purge. The desired thickness of the thin films is achieved by controlling the number of SVD cycles. In the case of Al\&LCys thin films, $6 \mathrm{~nm}$ thick was achieved after 50 SVD cycles and $12 \mathrm{~nm}$ thick was achieved after 50 SVD cycles with the expo mode. In the case of Al\&LAla thin films, $14 \mathrm{~nm}$ thick was achieved after 150 total SVD cycles. The substrates that were coated by the SVD include Si wafers, glass substrates, ACC (activated carbon cloth with 1,500 $\mathrm{m}^{2} / \mathrm{gr}$ surface area), carbon nanofibers (CNFs), and $\mathrm{HC}$ (hard carbon) particles.

\section{Surface characterization of the thin films}

The surfaces of the Al\&LCys thin films were characterized by a high-resolution scanning electron microscope (HR-SEM, FEI, Magellan 400L). The energy-dispersive X-ray analysis (EDAX) in the HR-SEM was executed by a $80 \mathrm{~mm}$ 2X-max detector (Oxford Instruments). Cross section images of the Al\&LCys thin films were taken using a Focused Ion Beam (FIB) Helios 600 scanning electron microscope system (FEI)

High magnification images of coated CNFs were taken by transmission electron microscope (TEM, JEM-1400, JEOL, LaB6, accelerating voltage $120 \mathrm{kV}$, bottom CCD 2x2k camera) and high-resolution transmission electron microscope (HR-TEM, JEM 2100, JEOL, 
Accelerating Voltage 200kV, Gatan USC 4000 4x4k camera). TEM samples were prepared on a lacey carbon-coated $\mathrm{Cu}$ grid.

Elemental analysis of the thin films and the chemical states of their surface were identified by (X-ray photoelectron spectroscopy) XPS in an Axis Ultra DLD system by Kratos Analytical using a monochromated Al-Ka X-ray beam, a hemispherical sector analyzer (HSA) and a multichannel detector. A pass energy of $20 \mathrm{eV}$, resulting in a full-width at half-maximum (FWHM) of less than $500 \mathrm{meV}$ for the $\mathrm{Ag}-3 \mathrm{~d} 5 / 2$ line, has been used. The spectra were calibrated in terms of charging-induced shifts by considering $\mathrm{C}-1 \mathrm{~s}$ peak (originating from carbon surface contamination) to be located at $284.6 \mathrm{eV}$. For the charging compensation, a low-energy electron gun (neutralizer) was used. The elemental analysis was performed using the Kratos-Vision software (Version 2.2.10) and its sensitivity factor database.

Atomic force microscope (AFM) images were performed using a dual microscope from Nanonics-Imaging which was freespace coupled to a LabRam HR Micro-Raman microscope from Horiba Jobin Yvon.

\section{Solid state NMR:}

${ }^{1} \mathrm{H}$ and ${ }^{27} \mathrm{Al}$ MAS NMR were conducted on a $200 \mathrm{MHz}$ Bruker Avance III spectrometer at room temperature, at spinning rates of 5-10 $\mathrm{kHz}$. The chemical shifts reported were referenced to TMS and $\mathrm{Al}\left(\mathrm{NO}_{3}\right)_{3(\mathrm{aq})}$. The $27 \mathrm{Al}$ spectra were collected using a rotorsynchronized solid echo pulse sequence, and the $1 \mathrm{H}$ spectra were collected with a rotorsynchronized Hahn echo sequence using variable delay times. Line deconvolutions were conducted using the TopSpin $3.5 \mathrm{pl} .7$ and DMfit40 software packages.

\section{Enantiospecifity chiral study of the thin films by CD spectroscopy:}

CD spectroscopy was used to study the chiral property of the thin films by the adsorption experiments of Phe as a chiral amino acid enantiomer. We chose to use Phe as the chiral probe molecule since it has a strong CD signal at a low concentration (even below 0.5 $\mathrm{mM}$ ). This system has already been proved as suitable for the study of chiral nanosized systems [ref]. Here, we study the adsorption of DL-Phe, D-Phe and L-Phe solutions onto the thin films. In a typical experiment, $2 \times 3 \mathrm{~cm}$ glass substrate or $14 \mathrm{~mm}$ diameter ACC coated with Al\&LCys (6 nm and $12 \mathrm{~nm}$ thick) or Al\&LAla (14 nm thick) were inserted into an aqueous solution of D-Phe, L-Phe and DL-Phe (5 mM, $41.3 \mathrm{mg}$ in $50 \mathrm{~mL})$ over 48 hours for reaching an equilibrium 
state with equal rates of adsorption and desorption. Evaluation of the $\mathrm{CD}$ adsorption measurements has clearly shown stereoselective adsorption of the enantiomers by the thin films, which display a significant difference in the adsorption and the CD ellipticity signals. The optical activity of the mother solutions was and the adsorbed solutions were measured by CD spectroscopy in the range of 205-245 $\mathrm{nm}$. The CD instrument of Chirascan CD spectrometer Applied Photophysics was used and set to a bandwidth of $1 \mathrm{~nm}$, a step size of 1 $\mathrm{nm}$, and a step time of $0.5 \mathrm{~s}$

\section{Enantiospecifity chiral study of the thin films crystallization Experiments:}

In a typical crystallization experiment, a supersaturated aqueous solutions of DL-Val (0.015 $\mathrm{mM}, 87.75 \mathrm{mg}$ in $50 \mathrm{~mL}$ water), DL- Glu (0.015 mM, $0.11025 \mathrm{gr}$ in $50 \mathrm{~mL}$ water), DL-His (0.015 mM, 0.116 gr in $50 \mathrm{~mL}$ water) and DL-Thr (0.015 mM, $0.893 \mathrm{mg}$ in $50 \mathrm{~mL}$ water) were separately prepared and stirred at $70{ }^{\circ} \mathrm{C}$ until fully dissolved. Then, a glass substrate with Al\&LCys (12 nm thick) or Al\&LAla (14 nm thick) thin films prepared over glass substrates $(2 \times 3 \mathrm{~cm})$ were placed in the as-prepared supersaturated DL- aqueous solution for 48 hours. Next, the crystals were dissolved in water and their optical rotation was studied by polarimetry. To compare, the optical rotation of the leftover solution was measured as well. These experiments were performed at room temperature, and the optical rotations were measured with a Jasco digital polarimeter (Model P-1010 $\mathrm{k}=586 \mathrm{~nm} \mathrm{a} \pm 0.05^{\circ}$ accuracy) using a cylindrical quartz cell $(5 \mathrm{~mL})$ at room temperature.

The phase of the crystals was studied by powder XRD using a Bruker AXS D8 advance diffractometer with $\mathrm{Cu} \mathrm{Ka}(\lambda=1.5418 \AA)$ operating at $40 \mathrm{kV} / 40 \mathrm{~mA}$, and collected from $2 \theta$ of $5^{\circ}$ to $50^{\circ}$. 